\title{
Laminar and turbulent comparisons for channel flow and flow control
}

\author{
By IVAN MARUSIC, D. D. JOSEPH \\ AND KRISHNAN MAHESH \\ Department of Aerospace Engineering and Mechanics, University of Minnesota, \\ Minneapolis, MN 55455, USA
}

(Received 3 May 2006 and in revised form 14 August 2006)

A formula is derived that shows exactly how much the discrepancy between the volume flux in laminar and in turbulent flow at the same pressure gradient increases as the pressure gradient is increased. We compare laminar and turbulent flows in channels with and without flow control. For the related problem of a fixed bulkReynolds-number flow, we seek the theoretical lowest bound for skin-friction drag for control schemes that use surface blowing and suction with zero-net volume-flux addition. For one such case, using a crossflow approach, we show that sustained drag below that of the laminar-Poiseuille-flow case is not possible. For more general control strategies we derive a criterion for achieving sublaminar drag and use this to consider the implications for control strategy design and the limitations at high Reynolds numbers.

\section{Introduction}

It is well known that in channels and pipes the volume flux of laminar flow is greater than that for turbulent flow at the same pressure gradient (Thomas 1942). Equivalently, the skin-friction drag in a laminar flow is less than in a turbulent flow for the same volume flux. In this paper we derive a formula to compute this volumeflux discrepancy for a given pressure gradient and consider the implications at high Reynolds numbers.

It has been conjectured (e.g. Bewley 2000) that, for constant volume-flux flows, sustained sublaminar drag is not possible even in the presence of flow control. Bewley considered zero-net volume-flux blowing or suction at the no-slip walls as a control strategy. (For the case of a fixed pressure gradient, the equivalent conjecture would be that such flow control cannot produce an average volume flux in excess of the laminar value.) However, several studies including Bewley \& Aamo (2004) and Cortelezzi et al. (1998), have demonstrated transient drag reductions below the laminar level. Recently, Min, Kang, Speyer \& Kim (2006) demonstrated that sustained sublaminar drag can in fact be achieved in a fully developed channel flow, albeit at a low Reynolds number. By 'sustained drag' we mean that the average value of the drag is independent of time. Min et al. (2006) used direct numerical simulations in an open-loop control strategy, with wall blowing and suction in the form of an upstream travelling wave, to achieve their result.

In this paper we consider laminar- and turbulent-flow comparisons for incompressible channel flows, with and without control, and discuss the implications for a range of Reynolds numbers. Two types of zero net-volume-flux blowing and 
suction control strategies are investigated. The first involves uniform-control flow across the channel, as was used by Fukagata, Iwamoto \& Kasagi (2002), and for this case we prove that sustained drag below that of the laminar-Poiseuille-flow case is not possible. The second control strategy addresses the more general case, with zero-mean flow at the walls, as was considered by Bewley \& Aamo (2004) and others. For this case we obtain a criterion for sustained sub-laminar conditions based on the power input of the control flow. Consideration of the criterion sheds light on why the control scheme of Min et al. (2006) was successful and indicates directions for modified control strategies that might yield further improvements.

\section{Equations for channel flow}

Much of the following extends the analyses presented by Busse (1970), Howard (1972) and Joseph (1974), who considered channel flows without control.

For a fully developed channel flow of an incompressible fluid, driven by a constant pressure gradient, the Navier-Stokes and continuity equations may be written as

$$
\begin{aligned}
\frac{\partial \boldsymbol{V}}{\partial t}+\boldsymbol{V} \cdot \nabla \boldsymbol{V} & =-\nabla p+\boldsymbol{e}_{x} P+\nabla^{2} \boldsymbol{V}, \\
\nabla \cdot \boldsymbol{V} & =0 .
\end{aligned}
$$

The Cartesian coordinate system used here defines $x$ and $y$ as the streamwise and spanwise directions respectively, and $z$ is the distance in the wall-normal direction from the centre of the channel. Unless indicated, here all terms have been nondimensionalized using $v$, the kinematic viscosity of the fluid, and $d$, the height of the channel. The domain occupied by the fluid is

$$
-\infty<x, y<\infty ;-1 / 2 \leqslant z \leqslant 1 / 2 .
$$

Here $P>0$ is the constant pressure gradient driving the flow, and the total pressure at a point in the fluid is

$$
p(x, y, z, t)-P x,
$$

where in all cases pressure has been normalized by $\rho$, the fluid density. We denote a wall-parallel plane average with an overbar:

$$
\bar{f}(z, t)=\lim _{L \rightarrow \infty} \frac{1}{(2 L)^{2}} \int_{-L}^{L} \int_{-L}^{L} f(x, y, z, t) \mathrm{d} x \mathrm{~d} y,
$$

and the overall average as

$$
\langle f\rangle=\int_{-1 / 2}^{1 / 2} \bar{f} \mathrm{~d} z
$$

Therefore, the Reynolds number $R e_{B}=\left\langle\bar{V}_{x}\right\rangle$ is that based on the channel height and the bulk velocity. We will also decompose the velocity and pressure into mean (wall-parallel plane-averaged) and fluctuating parts,

$$
\boldsymbol{V}=\overline{\boldsymbol{V}}+\boldsymbol{u}, \quad p=\bar{p}+p^{\prime},
$$

where the fluctuations have a zero mean: $\bar{u}, \bar{v}, \bar{w}, \bar{p}^{\prime}=0$.

\subsection{Boundary conditions}

We consider three types of channel flow. For all three cases

$$
V_{x}=V_{y}=0 \text { at } z= \pm 1 / 2
$$


while we have

for case $1, V_{z}=0$ at $z= \pm 1 / 2$;

for case $2, V_{z}=C(t)$ at $z= \pm 1 / 2$, where $\bar{C}=$ constant;

for case $3, V_{z}=\phi_{+}(x, y, t)$ at $z=1 / 2$ and $\phi_{-}(x, y, t)$ at $z=-1 / 2$, where $\bar{\phi}_{+}=$ $\bar{\phi}_{-}=0$.

Note that $u=v=0$ at $z \pm 1 / 2$ for all three cases, but $w=\phi=0$ for cases 1 and 2 only. Case 1 is conventional channel flow, while cases 2 and 3 cover the possible flows with blowing or suction at the walls and with zero net-volume-flux addition. For case 2, any flow introduced through the bottom wall is balanced by flow leaving the top wall. This flow was considered in control studies by Fukagata et al. (2002). Case 3 is the case considered by Bewley \& Aamo (2004), Min et al. (2006) and others; it allows for different functions for $V_{z}$ on the top and bottom walls provided that their mean is zero.

\subsection{Energy equations}

In the following we will make use of energy identities. These are derived first by substituting (2.3) into (2.1) and using continuity to give

$$
\frac{\partial \overline{\boldsymbol{V}}}{\partial t}+\frac{\partial \boldsymbol{u}}{\partial t}+\bar{V}_{z} \frac{\partial \overline{\boldsymbol{V}}}{\partial z}+\nabla \cdot(\overline{\boldsymbol{V}} \boldsymbol{u}+\boldsymbol{u} \overline{\boldsymbol{V}}+\boldsymbol{u} \boldsymbol{u})=-\boldsymbol{e}_{z} \frac{\partial \bar{p}}{\partial z}-\nabla p^{\prime}+\boldsymbol{e}_{x} P+\frac{\partial^{2} \overline{\boldsymbol{V}}}{\partial z^{2}}+\nabla^{2} \boldsymbol{u} .
$$

For cases 1 and $3, \bar{V}_{z}=0$ everywhere owing to (2.2), while for case $2, \bar{V}_{z}=C$. The wall-parallel plane average of (2.4) is

$$
\frac{\partial \overline{\boldsymbol{V}}}{\partial t}+\bar{V}_{z} \frac{\partial \overline{\boldsymbol{V}}}{\partial z}+\nabla \cdot(\overline{\boldsymbol{u u}})=-\boldsymbol{e}_{z} \frac{\partial \bar{p}}{\partial z}+\boldsymbol{e}_{x} P+\frac{\partial^{2} \overline{\boldsymbol{V}}}{\partial z^{2}},
$$

and the difference $(2.4)-(2.5)$ is

$$
\frac{\partial \boldsymbol{u}}{\partial t}+\nabla \cdot(\overline{\boldsymbol{V}} \boldsymbol{u}+\boldsymbol{u} \overline{\boldsymbol{V}}+\boldsymbol{u} \boldsymbol{u}-\overline{\boldsymbol{u} u})=-\nabla p^{\prime}+\nabla^{2} \boldsymbol{u} .
$$

An energy identity for the mean component is found by forming the dot product of $\overline{\boldsymbol{V}}$ with (2.5), taking the average and setting the result to zero, i.e. $\langle\overline{\boldsymbol{V}} \cdot(2.5)\rangle=0$, which gives

$$
\frac{1}{2} \frac{\mathrm{d}}{\mathrm{d} t}\left\langle|\overline{\boldsymbol{V}}|^{2}\right\rangle+\left\langle\bar{V}_{x} \frac{\partial \overline{u w}}{\partial z}+\bar{V}_{y} \frac{\partial \overline{v w}}{\partial z}\right\rangle=P\left\langle\bar{V}_{x}\right\rangle+\bar{V}_{z}[\bar{p}]_{\mp}-\left\langle\left|\frac{\partial \bar{V}_{x}}{\partial z}\right|^{2}+\left|\frac{\partial \bar{V}_{y}}{\partial z}\right|^{2}\right\rangle,
$$

where the notation []$_{\mp}$ indicates that the quantity at $z=1 / 2$ is subtracted from the quantity at $z=-1 / 2$, i.e. $[\bar{p}]_{\mp}=\bar{p}_{-}-\bar{p}_{+}$. The corresponding energy identity for the fluctuating parts is found from forming $\langle\boldsymbol{u} \cdot(2.6)\rangle=0$, which gives

$$
\frac{1}{2} \frac{\mathrm{d}}{\mathrm{d} t}\left\langle|\boldsymbol{u}|^{2}\right\rangle+\left\langle\overline{u w} \frac{\partial \bar{V}_{x}}{\partial z}+\overline{v w} \frac{\partial \bar{V}_{y}}{\partial z}\right\rangle=\Gamma-\left\langle|\nabla \boldsymbol{u}|^{2}\right\rangle
$$

where

$$
\Gamma=\left[\overline{\phi\left(p^{\prime}+\phi^{2} / 2\right)}\right]_{\mp} .
$$

(Here we note that $\boldsymbol{u} \cdot \partial \boldsymbol{u} / \partial z=0$ at the walls for all three cases. This can be shown by using Taylor series expansions, the continuity equation and the boundary conditions.)

Summing (2.7) and (2.8) gives the total energy equation

$$
\frac{1}{2} \frac{\mathrm{d}}{\mathrm{d} t}\left\langle|\overline{\boldsymbol{V}}|^{2}+|\boldsymbol{u}|^{2}\right\rangle=P\left\langle\bar{V}_{x}\right\rangle+\bar{V}_{z}[\bar{p}]_{\mp}+\Gamma-\left\langle|\nabla \boldsymbol{u}|^{2}+\left|\frac{\partial \bar{V}_{x}}{\partial z}\right|^{2}+\left|\frac{\partial \bar{V}_{y}}{\partial z}\right|^{2}\right\rangle .
$$


It may be noted that in addition to the driving pressure-gradient work term, $P\left\langle\bar{V}_{x}\right\rangle$, an extra energy-source term exists for case 2 owing to another pressure-gradient work term, $\bar{V}_{z}[\bar{p}]_{\mp}$, while for case 3 the extra term is $\Gamma$, which is due to the control flow at the walls and the associated induced fluctuating pressures.

\section{Volume-flux comparison between laminar and turbulent flows}

First we consider laminar Poiseuille flow (case 1 with $\boldsymbol{u}=0$ ). Here $\boldsymbol{V}=\left(U_{l}(z), 0,0\right)$ and (2.1) has the solution

for

$$
U_{l}(z)=\frac{3}{2}\left\langle U_{l}\right\rangle\left(1-4 z^{2}\right)
$$

$$
P_{l}=-\frac{\mathrm{d}^{2} U_{l}}{\mathrm{~d} z^{2}}=12\left\langle U_{l}\right\rangle
$$

In order to evaluate the bulk flow rate for the turbulent-flow case, $\left\langle\bar{V}_{x}\right\rangle$, we specify two properties of statistical stationarity. The first is that all wall-parallel plane averages, indicated by overbars, are time independent, and second we assume that velocity components have a zero mean value unless a non-zero mean value is forced externally. This latter property implies $\bar{V}_{y}=0$ for all flows considered here. Under such conditions (2.5) may be written as

$$
\frac{\mathrm{d}}{\mathrm{d} z}\left[\boldsymbol{e}_{x} \overline{V_{x} V_{z}}+\overline{\boldsymbol{u} w}+\boldsymbol{e}_{z} \bar{p}-\boldsymbol{e}_{x} P z-\frac{\mathrm{d} \overline{\boldsymbol{V}}}{\mathrm{d} z}\right]=0,
$$

where $\bar{V}_{x} V_{z}=\bar{V}_{x} \bar{V}_{z}$, since $\bar{V}_{z}=0$ or a constant. The energy equation (2.8) becomes

$$
-\left\langle\overline{u w} \frac{\mathrm{d} \bar{V}_{x}}{\mathrm{~d} z}\right\rangle+\Gamma=\left\langle|\nabla \boldsymbol{u}|^{2}\right\rangle .
$$

We now seek an expression for $P$ by taking the first integral of the $x$-component of (3.3):

$$
P z=\overline{u w}+\overline{V_{x} V_{z}}-\langle\overline{u w}\rangle-\left\langle\overline{V_{x} V_{z}}\right\rangle-\frac{\mathrm{d} \bar{V}_{x}}{\mathrm{~d} z} .
$$

The above equation is similar to the well-known linear relation for the stress. Forming $\langle z \cdot(3.5)\rangle=0$ gives

$$
P=12\left\langle z\left(\overline{u w}+\overline{V_{x} V_{z}}\right)\right\rangle+12\left\langle\bar{V}_{x}\right\rangle .
$$

The above equation is identical to that given by Fukugata et al. (2002) (equation (16) in their paper). A comparison between the volume flux in the channel for a turbulent flow with control and for the base laminar flow can now be made. For flows with the same driving pressure gradient $\left(P=P_{l}\right)$, using (3.6) and (3.2) the difference in the bulk flow rates for fully developed laminar flows and for turbulent flows is given by

$$
\left\langle U_{l}-\bar{V}_{x}\right\rangle=\left\langle z\left(\overline{u w}+\overline{V_{x} V_{z}}\right)\right\rangle .
$$

Therefore a proof that zero net-volume-flux blowing or suction control cannot produce a volume flux in excess of laminar flow requires

$$
\left\langle z\left(\overline{u w}+\overline{V_{x} V_{z}}\right)\right\rangle \geqslant 0 .
$$

To test this we form $\left\langle\left(\overline{u w}+\overline{V_{x} V_{z}}\right) \cdot(3.5)\right\rangle=0$ and using (3.4) obtain after some manipulation

$$
P\left\langle z\left(\overline{u w}+\overline{V_{x} V_{z}}\right)\right\rangle=\left\langle|\nabla \boldsymbol{u}|^{2}\right\rangle+\left\langle\left[\left(\overline{u w}+\overline{V_{x} V_{z}}\right)-\left(\langle\overline{u w}\rangle+\left\langle\overline{V_{x} V_{z}}\right\rangle\right)\right]^{2}\right\rangle-\Gamma .
$$




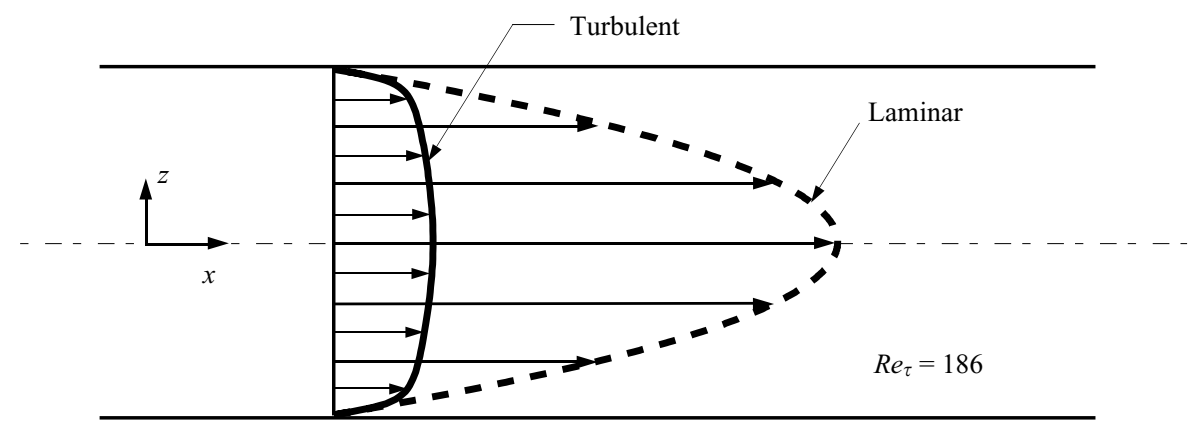

FiguRE 1. The turbulent mean-velocity profile, from the DNS dataset of del Álamo \& Jiménez (2003) at $R e_{\tau}=186$, and the corresponding laminar profile for the same driving pressure gradient. The profiles are drawn to scale (relative to each other).

Here $P>0$ by definition and, therefore, (3.9) indicates that (3.8) holds for case 2, since $\phi=0$ and hence $\Gamma=0$ and all other terms on the right-hand side are positive. However, for case 3 , where $\bar{V}_{z}=0$, the controlled flow can produce a volume flux in excess of laminar flow if and only if

$$
\Gamma>\left\langle|\nabla \boldsymbol{u}|^{2}\right\rangle+\left\langle[\overline{u w}-\langle\overline{u w}\rangle]^{2}\right\rangle .
$$

The same criterion holds for producing sublaminar drag, as will be discussed in $\S 4$. Although the drag is sublaminar, the power required to drive the flow may not be less than the laminar value (e.g. Choi, Moin \& Kim 1994, p. 79). Also, we note that the right-hand side of (3.10) will change with $\phi$, and the expression itself does not suggest a practical design strategy. However, it is clear that any control that yields sublaminar drag needs to satisfy (3.10) and that the specific term $\left[\overline{\phi\left(p^{\prime}+\phi^{2} / 2\right)}\right]_{\mp}$ needs to be positive. This will be further discussed in $\S 4.1$.

\subsection{Quantitative comparisons}

For regular channel flows and flows with crossflow control strategies (cases 1 and 2 in $\S 2.1$ ), the right-hand side of (3.7) is, as shown above, always positive, and hence the bulk flow rate for the laminar case is always higher for these flows. This is a more general statement of the 'mass-flux-discrepancy theorem' of Busse (1970) and Joseph (1974). A formal proof of this for regular pipe flow was first given by Thomas (1942). For regular channel flow, the result is well illustrated in figure 1, where a turbulent mean-velocity profile, taken from a direct numerical simulation (DNS) dataset at $R e_{\tau}=186$, together with, for comparison, the corresponding laminar profile (3.1) for the same level of driving pressure gradient. (Here $R e_{\tau}$ is the Kármán number, which is defined as the Reynolds number based on the channel half-width and the skin-friction velocity $\hat{U}_{\tau}=\left(\hat{\tau}_{0} / \rho\right)^{1 / 2}$.) The condition $P=P_{l}$ requires that the wall-normal gradients of the velocity profiles be equal at the walls.

While it is well known that the flow rate is higher in laminar than in turbulent flow at the same pressure gradient, we are not aware of any formula other than the one derived here from which the flow rates may be calculated. For example, the above results indicate that a laminar channel or pipe flow in transition to turbulence will always be associated with a bulk deceleration of the flow. Given the confined geometry, this transient event can only manifest itself through an adverse pressure gradient (i.e. one that is opposite to the driving pressure gradient). Few articles in the literature have reported this, an exception being a recent study by van Doorne (2004). Quantitative differences between the bulk flow rates (or volume fluxes) can be 


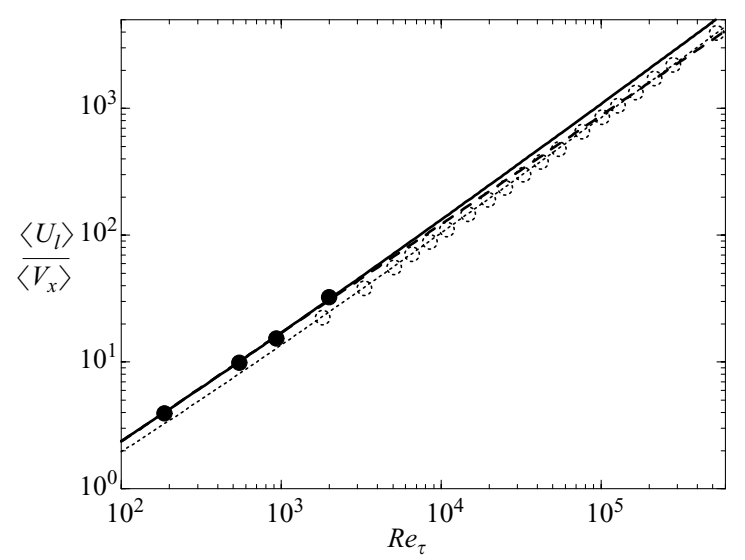

FIGURE 2. The ratio of the bulk velocities for laminar and turbulent channel flows as a function of $R e_{\tau}$. The solid line corresponds to (3.13). The dashed line corresponds to the same result but with the skin-friction formulae of Dean (1978). The solid circles are from the DNS datasets of del Álamo \& Jiménez (2003), del Álamo et al. (2004) and Hoyas \& Jiménez (2006). The $R e_{\tau}=186$ ordinate corresponds to the profiles in figure 1 . The dotted line corresponds to the expression for pipe flow. The dotted circles are from the pipe experiments of McKeon et al. (2004).

obtained using functional forms for the mean-velocity profiles (laminar and turbulent). For instance, using a law-of-the-wall-wake mean-velocity formulation, such as that given by Perry, Marusic \& Jones (2002) (their equation (4.6)) it can be shown that

$$
\frac{\left\langle\bar{V}_{x}\right\rangle-\bar{V}_{1}}{U_{\tau}} \approx-\frac{1}{\kappa}
$$

where $\bar{V}_{1}$ and $U_{\tau}$ are the non-dimensional centre-line and skin-friction velocities respectively and

$$
\frac{\bar{V}_{1}}{U_{\tau}}=\frac{1}{\kappa} \ln R e_{\tau}+A+\frac{1}{\kappa}\left(2 \Pi-\frac{1}{3}\right) ;
$$

here we take $\kappa=0.41, A=5.0$ and the Coles wake factor $\Pi=0.2$. From (3.2) we can show that the laminar bulk velocity $\left\langle U_{l}\right\rangle=2 R e_{\tau}^{2} / 3$, and using (3.7) for regular channel flows, with (3.11) and (3.12), we obtain

$$
\left\langle U_{l}-\bar{V}_{x}\right\rangle=\langle z \overline{u w}\rangle=\frac{2}{3} R e_{\tau}^{2}-\frac{2}{\kappa} R e_{\tau}\left(\ln R e_{\tau}+C\right),
$$

where $P=8 R e_{\tau}^{2}$ and $C=\kappa A+2 \Pi-4 / 3=1.117$, using the values given above.

Figure 2 shows for comparison the bulk velocities for laminar and turbulent flows for varying levels of $R e_{\tau}$. The solid line is obtained using the above expressions for channel flows. For a typical practical Reynolds number $\operatorname{Re}_{\tau}=10^{5}$ the ratio $\left\langle U_{l}\right\rangle /\left\langle\bar{V}_{x}\right\rangle$ is seen to be over $1000 . \dagger$ The ratio of the laminar and turbulent velocities is also a convenient way of illustrating how $\langle z \overline{u w}\rangle$ changes with $R e_{\tau}$, and a corresponding plot is shown in figure 3; the asymptotic trend is equivalent to the condition that the

$\dagger$ This example is in the spirit of A. N. Kolmogorov who, as explained by Barenblatt (2005), would start his course on turbulence at Moscow State University by asking the students what the flow rate of the Volga river would be if by some miracle it became laminar. The answer is striking, with surface velocities in the hundreds of kilometres per second. 


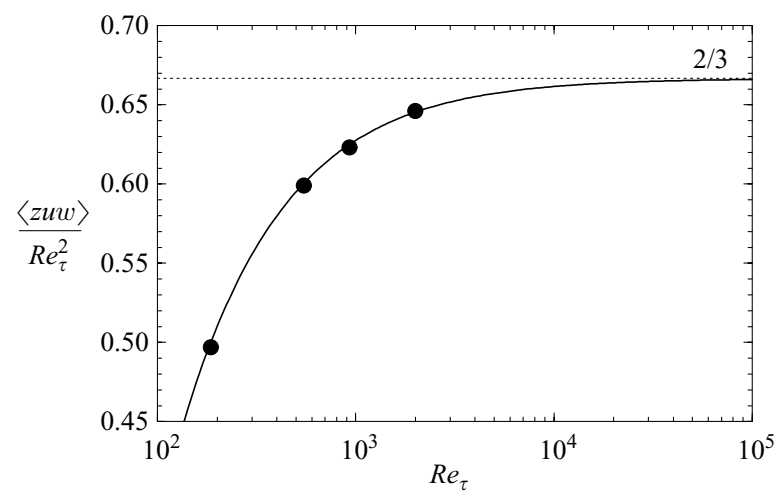

FIgURE 3. Formulation for $\langle z \overline{u w}\rangle$ (using (3.13)) as a function of $R e_{\tau}$. The solid circles correspond to the DNS data as in figure 2.

Reynolds shear stress $\overline{u w}$ approaches a linear function as $R e_{B} \rightarrow \infty$, in which case it directly follows that $\langle z \overline{u w}\rangle \rightarrow 2 R e_{\tau}^{2} / 3$. As in figure 2 , the formulation is seen to agree very well with data obtained from direct numerical simulations (DNSs), where $\langle z \overline{u w}\rangle$ values are computed directly.

Similar agreement can be obtained using other formulas in the literature. For example, the dashed line in figure 2 is obtained using $C_{f}=0.073 R e_{B}^{-1 / 4}$ and $C_{f_{l}}=$ $12 / R e_{B}$ from Dean (1978). The present authors prefer using log-law-based expressions such as (3.12), from which Dean's formula deviates at high $R e_{\tau}$. Also shown in the figure is the corresponding curve for pipe flow, obtained using (3.12) with the same constants, which is seen to agree very well with the experimental data of McKeon et al. (2004) obtained in the Princeton superpipe across a large range of Reynolds number. (Here for pipe flow $\left\langle U_{l}\right\rangle=R e_{\tau}^{2} / 2$ and $\left\langle\bar{V}_{x}\right\rangle=2 R e_{\tau}\left(\bar{V}_{1} / U_{\tau}-3 / 2 \kappa\right)$. More refined log-law based expressions are given in McKeon, Zagarola \& Smits 2005.)

\section{Drag comparisons}

For a fully developed flow with statistical stationarity, the net skin-friction drag is simply obtained from a balance with the pressure gradient forces. That is,

$$
2 \hat{\tau}_{0}\left(4 L^{2}\right)=\rho \hat{P}\left(4 L^{2} d\right)
$$

where $\hat{\tau}_{0}$ and $\hat{P}$ are the dimensional average wall-shear stress and the driving pressure gradient respectively. From this it follows that the bulk skin-friction coefficient $C_{f}=$ $2 \hat{\tau}_{0} /\left(\rho \hat{U}_{B}^{2}\right)$, where $\hat{U}_{B}$ is the dimensional bulk velocity, is related to $P$ by

$$
C_{f}=\frac{P}{R e_{B}^{2}} .
$$

Using (3.6), (3.2) and (4.1) for a given Reynolds number, $\left\langle U_{l}\right\rangle=\left\langle\bar{V}_{x}\right\rangle=R e_{B}$, we obtain

$$
C_{f}-C_{f_{l}}=\frac{12}{R e_{B}^{2}}\left\langle z\left(\overline{u w}+\overline{V_{x} V_{z}}\right)\right\rangle .
$$

Equation (4.2) is equivalent to the result obtained by Fukagata et al. (2002), according to which the drag reduction is dependent on the weighted integral of the Reynolds shear stress. Bewley \& Aamo (2004) derived a related quantity for $\bar{V}_{z}=0$ (case 3). 
As expected, the criterion for achieving sublaminar drag with control for a fixed volume flux is equivalent to exceeding the volume flux of laminar flow for a fixed pressure gradient. Both depend on $\left\langle z\left(\overline{u w}+\overline{V_{x} V_{z}}\right)\right\rangle$. Therefore, from (3.7) and (3.9) we may also conclude that for regular channel flows and flows with crossflow control strategies (cases 1 and 2)

$$
C_{f}-C_{f_{l}} \geqslant 0 .
$$

For case 3, where $\bar{V}_{z}=0,(3.10)$ holds and therefore the controlled flow can produce sustained sublaminar skin-friction levels if and only if

$$
\Gamma>\left\langle|\nabla \boldsymbol{u}|^{2}\right\rangle+\left\langle[\overline{u w}-\langle\overline{u w}\rangle]^{2}\right\rangle .
$$

\subsection{Implications for control strategies}

Equation (3.10) gives the criterion for achieving sustained sublaminar drag; from it we may identify the relevant control parameter $\Gamma=\left[\overline{\phi\left(p^{\prime}+\phi^{2} / 2\right)}\right]_{\mp}$, which is solely defined on the walls of the channel. The criterion does not directly yield a practical design strategy but does provide insights into strategies that have been proposed in the literature.

In (3.10), $\Gamma$ relates to the power input of the control signal, and as expected increasing $\Gamma$ decreases the net skin-friction drag. However, it may be noted that maximizing $\Gamma$ requires a control strategy that sums the contributions from the opposing walls. Therefore, in order to consider a physical interpretation for $\Gamma$ we restrict our attention to the bottom wall in flows with symmetric actuation. In this case we require that $\left[\overline{\phi\left(p^{\prime}+\phi^{2} / 2\right)}\right]_{-}$be positive. For any periodic blowing and suction (as was used by Min et al. 2006) $\phi^{3}$ yields zero when averaged, and therefore the only contribution to $\Gamma$ can come from $p^{\prime} \phi$. We therefore require that blowing (positive $\phi$ ) be accompanied by an increase in pressure, while suction (negative $\phi$ ) be accompanied by a decrease in pressure at the wall.

It is known from studies on jets in crossflow (e.g. Muppidi \& Mahesh 2005) that a crossflowing jet yields high levels of pressure, especially at the leading edge of the jet; i.e. a positive value of $\phi$ yields positive values of $p^{\prime}$. Similarly, high levels of suction will modify the local flow to resemble a point sink. The external flow accelerates toward the source of suction, in order to conserve mass; the acceleration sets up a favourable pressure gradient in the direction of the suction and therefore decreases the pressure at the source of suction. Negative values of $\phi$ therefore produce negative values of $p^{\prime}$ and so the resulting product is positive.

To test this interpretation, direct numerical simulations were carried out for idealized flows with blowing and suction into a cross-stream, using the numerical approach as described by Muppidi \& Mahesh (2005). The geometry is that of a twodimensional channel with dimensions $20 \times 10 \times 1$ per unit slot width, in the streamwise, wall-normal and spanwise directions respectively. The width of the slot extends over the entire span, over which periodic conditions are imposed. Free-stream Dirichlet conditions are prescribed at the top boundary, and convective boundary conditions are prescribed at the outflow. Note that the domain height is large enough not to affect the solution. A top-hat profile is prescribed for the jet through the slot, and a uniform crossflow is prescribed at the inflow. Along the bottom wall, the uniform crossflow forms a boundary layer whose thickness with respect to the jet is small. The simulation results therefore depend essentially upon the parameter $r s$, where $r$ denotes the velocity ratio of the jet and the crossflow and $s$ denotes the slot width.

While these flows are simplified, the general trends are expected to hold very near the blowing and suction ports since $\Gamma$ is a quantity evaluated exactly at the wall. 

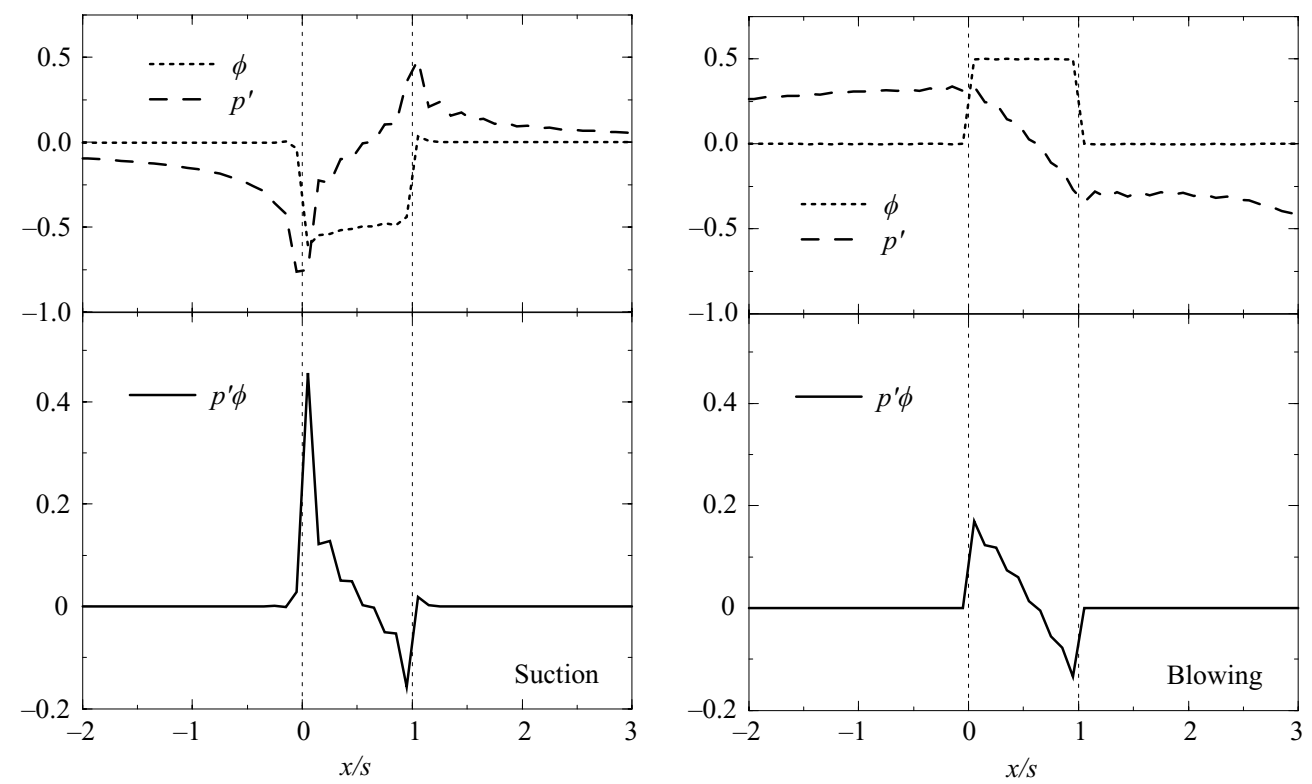

FiguRE 4. Numerical-simulation results for two-dimensional blowing and suction into a crossflow, moving from right to left. The blowing and suction flows are initially applied uniformly across $0 \leqslant x / s \leqslant 1$. The wall velocities and pressures here are normalized by the crossflow velocity. Note that the average value of $\overline{p^{\prime} \phi}$ on the wall for both blowing and suction is positive.

The results are shown in figure 4 where, for both blowing and suction, $\overline{p^{\prime} \phi}$ is found to be positive provided that the level of blowing and suction is sufficiently high. For instance, in figure 4 the ratio of $\phi$ and the crossflow speed was 0.5 , while a ratio of 0.1 was found to be insufficient to produce a positive $\overline{p^{\prime} \phi}$ value.

The above mechanism for generating high $\overline{p^{\prime} \phi}$ values on the bottom wall is consistent with the approach used by Min et al. (2006), who demonstrated sublaminar drag using a travelling wave blowing and suction strategy with

$$
\phi=a \cos [\alpha(x-c t)],
$$

where $c$ is the wave speed. Relatively high levels of blowing and suction were required by Min et al. (2006) to achieve sublaminar drag combined with upstream travelling waves ( $c$ negative). The control flow was also implemented on both walls in varicose mode, i.e. the upper and lower walls have blowing and suction in phase at the same streamwise location. This symmetric actuation is consistent with adding the effects from the top and bottom walls while an upward-travelling wave increases the relative velocity between the jet and the crossflow, which means that for the same jet velocity $\phi$, the stagnation pressure $p^{\prime}$ at the leading edge of the jet would be higher; i.e. the upward-traveling wave generates higher positive levels of $p^{\prime} \phi$. Conversely, a downward-traveling wave would, for small phase speeds, decrease the relative velocity and thereby decrease the stagnation pressure at the jet leading edge. The resulting value of $p^{\prime} \phi$ would be smaller and therefore less effective in reducing drag. If the downstream wave speed is large enough, then the crossflow would have to accelerate in the streamwise direction to occupy the region evacuated by the jet; the acceleration would decrease the pressure in the vicinity of the jet, i.e. $p^{\prime} \phi$ would be negative, and 
the drag would actually increase. This is consistent with what Min et al. (2006) found for positive values of $c$.

Considering (3.10) and the expression for $\Gamma$ it would seem that the control strategy used by Min et al. (2006) could perhaps be further improved by modifying the control to yield positive and negative values of $\overline{\phi^{3}}$ on the bottom and top walls respectively. This would require that the input control flow be skewed. Since from the results in figure 4 it appears that suction has a higher contribution to $\overline{p^{\prime} \phi}$ than does blowing, this would indicate that possibly using a symmetric varicose blowing and suction upstream-travelling waveform that is biased towards more intense suction (while still maintaining $\bar{\phi}=0$ ) might be beneficial.

\section{Concluding remarks}

For turbulence control, it is necessary to understand how to achieve sublaminar levels of drag at high Reynolds numbers. For the general blowing and suction control strategy (case 3 ) this relates directly to $\langle z \overline{u w}\rangle$. Figure 3 shows that $\langle z \overline{u w}\rangle$ has very high values at high Reynolds numbers (since it increases nominally with $R e_{\tau}^{2}$ ), which makes relaminarization or sublaminar drag reduction by control difficult. Min et al. (2006) achieved sublaminar conditions at $R e_{\tau} \approx 120$, but at high Reynolds numbers figure 2 shows that $\langle z \overline{u w}\rangle$ would be many orders of magnitude higher. However, a control scheme need not relaminarize a flow from a high Reynolds number but could instead modify the Reynolds shear-stress profile in such a way as to minimize the weighted integral $\langle z \overline{u w}\rangle$. Iwamoto et al. (2005) used this strategy recently and showed that small changes to $\overline{u w}$ in the near-wall region at high Reynolds number can lead to substantial reductions in skin friction.

Comparison of the volume flux for laminar and turbulent flows for a given pressure gradient also highlights that, in general, reducing the turbulence level in flows driven by a steady energy source can lead to significant increases of mean velocity. Another example of this, which in the authors' opinion is worth mentioning, relates to the modification of turbulence by the transport of small particles (Gore \& Crowe 1989). Barenblatt, Chorin \& Prostokishin (2005) considered such a mechanism to explain why accelerated levels of wind speed exist in tropical cyclones in regions near the water surface, where ocean spray droplets reside. Here it is believed that the turbulence energy that goes into suspending the particles in the flow leads to a reduction in the flow turbulence intensity and consequently dramatic increases in the mean wind speed. Similar mechanisms may explain why sand storms are typically associated with high sustained wind speeds, which allow the storm to travel over very long distances.

This work was in part supported by the National Science Foundation (IM with CTS-0324898, DDJ with CTS-0302837 and KM with CTS-0133837), and the David and Lucile Packard Foundation. We are also grateful to Suman Muppidi for useful discussions.

\section{REFERENCES}

Del Álamo, J. C. \& Jiménez, J. 2003 Spectra of the very large anisotropic scales in turbulent channels. Phys. Fluids 15(6), L41-L44.

del Álamo, J. C., Jiménez, J., Zandonade, P. \& Moser, R. D. 2004 Scaling of the energy spectra of turbulent channels. J. Fluid Mech. 500, 135-144.

Barenblatt, G. I. 2005 Applied mechanics: an age old science perpetually in rebirth. ASME Timoshenko Medal Acceptance Speech. 
Barenblatt, G. I., Chorin, A. J. \& Prostokishin, V. M. 2005 A note concerning the Lighthill 'sandwich model' of tropical cyclones. Proc. Natl Acad. Sci. 102, 11148-11150.

Bewley, T. R. 2001 Flow control: new challenges for a new renaissance. Prog. Aerospace Sci. 37, 21.

Bewley, T. R. \& Aamo, O. M. 2004 A 'win-win' mechanism for low-drag transients in controlled two-dimensional channel flow and its implications for sustained drag reduction. J. Fluid Mech. 499, 183-196.

Busse, F. H. 1970 Bounds for turbulent shear flow. J. Fluid Mech. 41, 219-240.

Chol, H., Morn, P. \& Kim, J. 1994 Active turbulence control for drag reduction in wall-bounded flows. J. Fluid Mech. 262, 75-110.

Cortelezzi, L., Lee, K. H., Kim, J. \& Speyer, J. L. 1998 Skin-friction drag reduction via robust reduced-order linear feedback control. Intl J. Comput. Fluid Dyn. 11, 79-92.

DEAN, R. B. 1978 Reynolds number dependence of skin friction and other bulk flow variables in two-dimensional rectangular duct flow. Trans. ASME: J. Fluids Engng 100, 215-223.

VAN Doorne, C. W. H. 2004 Stereoscopic PIV on transition in pipe flow. PhD thesis, TU-Delft, Netherlands.

Fukagata, K., Iwamoto, K. \& Kasagi, N. 2002 Contribution of Reynolds stress distribution to the skin friction in wall-bounded flows. Phys. Fluids 14(11), L73-L76.

Gore, R. \& Crowe, C. 1989 Effect of particle size on modulating turbulent intensity. Intl J. Multiphase Flow 15, 279-285.

Howard, L. N. 1972 Bounds on flow quantities. Annu. Rev. Fluid Mech. 4, 473-494.

Hoyas, S. \& JimÉnez, J. 2006 Scaling of the velocity fluctuations in turbulent channels up to $R e_{\tau}=2003$. Phys. Fluids 18, 011702.

Iwamoto, K., Fukagata, K., Kasagi, N. \& Suzuki, Y. 2005 Friction drag reduction achievable by near-wall turbulence manipulation at high Reynolds number. Phys. Fluids 17, 011702.

Joseph, D. D. 1974 Response curves for plane Poiseuille flow. Adv. Appli. Mech. 14, 241-278.

McKeon, B., Li, J., Jiang, W., Morrison, J. \& Smits, A. 2004 Further observations on the mean velocity in fully-developed pipe flow. J. Fluid Mech. 501, 135-147.

McKeon, B., Zagarola, M. \& Smits, A. 2005 A new friction factor relationship for fully developed pipe flow. J. Fluid Mech. 538, 429-443.

Min, T., KAng, S., Speyer, J. L. \& Kim, J. 2006 Sustained sub-laminar drag in a fully developed channel flow. J. Fluid Mech. 558, 309-318.

MupPIDI, S. \& MAHeSh, K. 2005 Study of trajectories of jets in crossflow using direct numerical simulations. J. Fluid Mech. 530, 81-100.

Perry, A. E., Marusic, I. \& Jones, M. B. 2002 On the streamwise evolution of turbulent boundary layers in arbitrary pressure gradients. J. Fluid Mech. 461, 61-91.

Thomas, T. Y. 1942 Qualitative analysis of the flow of fluids in pipes. Am. J. Maths 64, 754-767. 


\section{University Library}

\section{- M M I N E R VA A gateway to Melbourne's research publications}

Minerva Access is the Institutional Repository of The University of Melbourne

Author/s:

Marusic, I;Joseph, DD;Mahesh, K

Title:

Laminar and turbulent comparisons for channel flow and flow control

Date:

2007-01-10

Citation:

Marusic, I., Joseph, D. D. \& Mahesh, K. (2007). Laminar and turbulent comparisons for channel flow and flow control. JOURNAL OF FLUID MECHANICS, 570, pp.467-477. https:// doi.org/10.1017/S0022112006003247.

Publication Status:

Published

Persistent Link:

http://hdl.handle.net/11343/33538 